IUFM de Lyon, ICAR, UMR CNRS 5191, Université Lyon 2

\title{
L'effacement énonciatif dans les discours rapportés et ses effets pragmatiques
}

Depuis le $\mathrm{n}^{\circ} 117$ de Langages consacré aux analyses du discours en France, ce courant n'a cessé de se développer en diversifiant les études de corpus et en approfondissant ses outils, ainsi qu'en témoignent les dictionnaires récents de Détrie, Siblot et Verine 2001 ou de Charaudeau et Maingueneau 2002. Ce numéro apporte sa contribution à ce mouvement en revenant sur la question des discours rapportés, avec l'objectif prioritaire d'examiner les effets pragmatiques de l'effacement énonciatif (EE) sur la construction interactionnelle des points de vue, notamment les phénomènes de surénonciation et de sousénonciation, qui, avec la coénonciation, structurent (ce qu'on propose de nommer) la topique énonciative. Compte tenu du caractère programmatique de la problématique, cette introduction présentera les articles du numéro en les inscrivant dans un cadre de propositions solidaires, depuis les fondations théoriques de $l^{\prime} \mathrm{EE}^{1}$, ses spécificités lorsqu'il s'agit de rapporter des discours, jusqu'aux effets en termes de prise en charge énonciative.

\section{EFFACEMENT ÉNONCIATIF STRICTO SENSU ET DÉSINSCRIPTION ÉNONCIATIVE}

L'EE - si l'on cherche à le définir en référence au concept de Benveniste d'appareil formel de l'énonciation - correspond non à un " "appareil formel" ${ }^{2}$ de la non-énonciation » (car acte d'énonciation il y a), mais à un « "appareil

1. Les contraintes éditoriales nous font faire l'impasse sur les travaux antérieurs qui ont abordé la question de l'EE, tant dans un cadre énonciatif (indiciel) que pragmatique ou interdiscursif (polyphonie, dialogisme), dont on trouve maintes traces dans Langages ou Langue française. Si ce numéro prétend à une certaine originalité, c'est dans la convergence des trois cadres théoriques précédents.

2. Si tant est que l'expression soit adéquate. 
formel" de l'effacement énonciatif », le locuteur donnant l'impression " qu'il se retire de l'énonciation, qu'il "objectivise" son discours en "gommant" non seulement les marques les plus manifestes de sa présence (les embrayeurs) mais également le marquage de toute source énonciative identifiable » (Vion 2001 : 334). Vion exemplifie cette stratégie par les plans d'énonciation historique ou théorique qui laissent penser que les événements ou les arguments présentés sont indépendants de toute intervention du sujet parlant. Cette idée de " simulacre énonciatif »se retrouve également chez Charaudeau, lorsqu'il évoque " un "jeu" que joue le sujet parlant, comme s'il lui était possible de ne pas avoir de point de vue, de disparaître complètement de l'acte d'énonciation, et de laisser parler le discours par lui-même »(Charaudeau 1992 : 650).

Relèvent de l'EE les énoncés désembrayés (énonciation historique et théorique), à la condition que les repérages anaphoriques s'accompagnent du moins de marques de subjectivité possible, afin que la référenciation des objets de discours paraisse quasiment indépendante de la situation d'énonciation du locuteur comme de sa subjectivité ; néanmoins, si ces énoncés désembrayés s'accompagnent de nombreux subjectivèmes, ils ne sont que marginalement concernés par l'EE. Réciproquement, si un énoncé embrayé ne relève pas de l'EE, néanmoins, dès qu'il comporte peu de marques de subjectivité et que les marqueurs personnels et spatio-temporels sont vidés de toute référence pertinente à leur contexte de production, alors l'énonciation personnelle tend vers $\mathrm{l}^{\prime} \mathrm{EE}$, du fait de cette désinscription énonciative. L'EE apparaît ainsi comme un phénomène graduel, minimal avec le phénomène de désinscription énonciative, maximal avec les plans d'énonciation non embrayés (historique et théorique) " objectivants ».

L'objectivation de l'objet et l'effacement de l'énonciateur (mais non du locuteur, $c f$. infra) se marquent sur les plans discursif (mises en description dynamisant les objets, narration hétérodiégétique avec narrateur anonyme, argumentation avec énonciateur générique...), rhétorique et stylistique (personnification, vitalisation, effets de montage, de liste...). Sur le plan linguistique, $l^{\prime} E E$ est signalé, outre les plans d'énonciation historique et théorique, par un ensemble de marques affectant la référence nominale et verbale. Ainsi, au plan de la référence nominale, des nominalisations, du rôle des SN précédés de déterminants à valeur générique, de l'emploi de termes " neutres ", non marqués, paraissant échapper à la problématique de la connotation, des pronoms indéfinis. Ainsi encore, au plan de la référence verbale, des phrases averbales, des phrases simples, des construction paratactiques (évitant autant que faire se peut les valeurs énonciatives associées aux conjonctions et locutions conjonctives), des infinitivations, impersonnalisations, passivations, accompagnant les énoncés génériques, stéréotypiques ou doxiques...

\section{DISJONCTION LOCUTEUR/ÉNONCIATEUR}

Chez Benveniste 1966 et 1974, locuteur et énonciateur sont synonymes, subjectivité et énonciation personnelle, coextensives (cf. Philippe 2002). Cette 


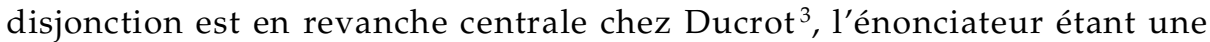
instance intradiscursive à la source du point de vue $(\mathrm{pdv})$ contenu dans un contenu propositionnel. Dans cette conception polyphonique, le locuteur est un metteur en scène qui organise la régie entre des énonciateurs variés (Ducrot 1984 : 204-205). Cette disjonction repose sur le fait que, si tout locuteur est aussi énonciateur (en vertu du principe de sincérité), en revanche, tout énonciateur n'est pas nécessairement locuteur. C'est ce que montre le DIL, qui, loin d'être un effet de style à la limite du système, exhibe des potentialités découlant du dialogisme de la langue ${ }^{4}$ (Vion 1998, Charaudeau et Maingueneau 2002 : 226, Rabatel 2003d) : ainsi des énoncés ironiques, doxiques ou des points de vue narratifs $\left(\mathrm{PDV}^{5}\right)$ comme en (1) :

(1) Jeanne, ayant fini ses malles, s'approcha de la fenêtre, mais la pluie ne cessait pas (Ducrot 1980a : 20)

Ducrot remarque que même si Jeanne n'est pas à l'origine d'une parole, l'énoncé, raconté par le locuteur premier (le narrateur), envisage les faits du PDV de Jeanne, motivant le mouvement vers la fenêtre par son impatience, la référenciation indiquant que Jeanne - qui est ainsi énonciateur, sans être locuteur ${ }^{6}$ - est déçue ( $c f$. " mais », le sémantisme du verbe « cesser », la négation, etc.) :

Le locuteur, responsable de l'énoncé, donne existence, au moyen de celui-ci, à des énonciateurs dont il organise les points de vue et les attitudes. Et sa position propre peut se manifester soit parce qu'il s'assimile à tel ou tel des énonciateurs, en le prenant pour représentant (l'énonciateur est alors actualisé), soit simplement parce

3. Elle est en germe chez Bally, Guillaume, Damourette et Pichon ; on la retrouve chez AuthierRevuz 1995, autour de la notion d'hétérogénéité.

4. On oppose souvent le " dialogisme de Bakhtine " à la " polyphonie de Ducrot ", en faisant de la seconde, davantage centrée sur le marquage linguistique des voix (hétérogénéité montrée), un sous-ensemble du premier, davantage traversé par l'interdiscours. Cette opposition est à relativiser, puisque, à lire Bres 2001 : 87, les marques du dialogisme sont les mêmes que celles qui sont citées par Nølke 2002 : 447 pour la polyphonie (excepté ce qui relève du dialogisme de la nomination). À la différence de la polyphonie, le dialogisme accorde une large place à la dimension interdiscursive (dialogismes interlocutif et interdiscursif, autodialogisme), en un sens proche de l'hétérogénéité constitutive et montrée (Authier-Revuz 1995). Quant à l'opposition entre une égalité des voix (polyphonie) et hiérarchisation (dialogisme), elle mêle l'ordre narratologique, avec l'égalité des voix entre personnages et narrateur ( $c f$. Dostoïevski) et l'ordre linguistique, où la hiérarchisation est la règle.

5. Les majuscules indiquent que notre conception du point de vue diffère de celle de Ducrot. Tout peut faire PDV, pour autant que la référenciation oriente l'interprétation dans une certaine direction. Point n'est donc besoin qu'un PDV exprime explicitement une opinion ou une thèse, il suffit qu'un objet soit ainsi dénoté que cela renseigne sur le PDV du locuteur quant à l'objet et influe sur l'interprétation du destinataire, $c f$. infra.

6. Les relations entre locuteur premier (noté L1) et cet énonciateur second (e2) relèvent de la responsabilité ou de la non-responsabilité, lorsque les relations sont explicites, de la consonance ou de la dissonance (Cohn 1981), lorsque ces liens sont implicites. Dans les deux cas, ces liens sont graduels. En l'occurrence, en l'absence de marque explicite, et compte tenu du pacte de croyance du récit réaliste, il y a consonance entre le point de vue de Jeanne et celui du narrateur, qui l'accrédite comme vrai, puisqu'il ne marque pas de dissonance ou de distance. 
qu'il a choisi de les faire apparaître et que leur apparition reste significative, même s'il ne s'assimile pas à eux (Ducrot 1984 : 205).

J'appelle " énonciateurs " ces êtres qui sont censés s'exprimer à travers l'énonciation, sans que pour autant on leur attribue des mots précis ; s'ils " parlent », c'est seulement en ce sens que l'énonciation est vue comme exprimant leur point de vue, leur position, leur attitude, mais non pas, au sens matériel du terme, leurs paroles (Ducrot 1984 : 204).

Ducrot définit solidairement l'énonciateur et le pdv. Toutefois, derrière l'implication réciproque des termes (pas de pdv sans énonciateur, pas d'énonciateur sans pdv), les deux concepts ne fonctionnent pas au même niveau, la notion de pdv servant à définir l'énonciateur (disjoint du locuteur) comme la source d'un contenu propositionnel exprimant un pdv - ce dernier ne se manifestant pas que dans le modus, mais aussi dans le dictum (Ducrot 1993 : 128).

On distinguera donc le locuteur (instance qui profère un énoncé embrayé ou désembrayé, dans ses dimensions phonétiques et phatiques ou scripturales) de l'énonciateur (instance des actualisations opérées par le sujet modal) qui assume l'énoncé en un sens nettement moins abstrait que la prise en charge découlant de l'ancrage déictique (Rabatel 2004c). Une telle distinction, qui $\mathrm{n}^{\prime}$ exclut pas les syncrétismes ${ }^{7}$ fréquents entre locuteur et énonciateur - ou entre locuteurs en tant que tel et en tant qu'être du monde -, permet de rendre compte des déséquilibres interactionnels, notamment en raison du pouvoir accordé aux énonciateurs. Sur ce point, il y a débat avec Ducrot 1984 : 153 et 215 selon qui l'énonciateur ne peut effectuer des actes de langage, à la différence du locuteur. Cette conception se discute, lorsque les contenus propositionnels ne se limitent pas à leur dimension constative. C'est le cas de (1) : cet énoncé descriptif exprime indirectement un vif souhait de sortir. Seule la saisie de ce souhait fait interpréter correctement ce PDV, sur la base d'une saturation sémantique du sujet modal : Jeanne ne se réduit pas à un pur centre scopique, elle est représentée comme un sujet praxéologique, du fait de la référenciation de sa perception.

Autre différence avec Ducrot : plutôt que de considérer qu'il y a autant d'énonciateurs que de contenus propositionnels, il paraît cognitivement moins coûteux de regrouper ceux qui peuvent avoir des contenus thématiques divers, mais que rassemblent la même origine et la même visée énonciative, surtout si les énonciateurs sont incarnés ${ }^{8}$ (Rabatel 2004d, i). Sur le plan pragmatique, enfin, cela (re)pose la question de la hiérarchisation des énonciateurs et des relations entre énonciateurs et locuteurs. Question sensible, tant il y a des inconvénients à alimenter la thèse d'un locuteur abstrait et quasi fantasmagorique, grand

7. Notés par une barre oblique, L1/E1 pour le syncrétisme du locuteur/énonciateur premier, ou 12/e2 pour le locuteur/énonciateur second, les minuscules indiquant le caractère dominé du second, sur le plan des repérages et des effets pragmatiques.

8. Ce choix entraîne la prise en compte de la saturation sémantique des énonciateurs, variable selon leur degré d'incarnation, comme le signalent les théoriciens de la théorie SCAndinave de la Polyphonie LINguistiquE (scapoline) avec les « êtres discursifs » (Nølke et Olsen 2000 : 50). 
metteur en scène qui se jouerait des rôles énonciatifs pour éviter d'être assigné à une position qu'il faudrait assumer devant les autres. La survalorisation du locuteur en tant que tel, au détriment du locuteur être du monde ${ }^{9}$, pose problème car elle alimente les représentations idéalistes de " sa majesté le "sujet" », qui perdurent derrière le paravent de la polyphonie. C'est le reproche $q^{\prime}$ 'Authier-Revuz adresse à toutes les métaphores (" théâtre ", " mise en scène », " jeux de rôle ») qui concourent à la représentation d'un sujet tout puissant à travers les manipulations de la communication, au déni du sujet toujours décentré, clivé, divisé, y compris dans son illusoire position de maîtrise, dont témoigne l'hétérogénéité de sa parole (Authier-Revuz 1998 : 71) ${ }^{10}$.

D'où la proposition d'accorder une importance toute particulière à l'énonciateur en syncrétisme avec le locuteur en tant que tel et en tant qu'être du monde (voire avec le sujet parlant), c'est-à-dire à celui par rapport auquel les interactants se situent (à qui ils sont en droit de demander des comptes, le cas échéant) et dont le PDV se trouve constamment (re)paramétré, pour ainsi dire, par les multiples modalités de circulation des discours, qui donnent naissance à des PDV surplombants, partagés ou dominés (cf. infra, 4). À terme, l'objectif n'est pas de réintroduire subrepticement l'unicité du sujet derrière la multiplicité des rôles, mais de penser les contradictions, les conflits, dans un cadre interactionnel en perpétuelle recomposition.

\section{EFFACEMENT ÉNONCIATIF ET DISCOURS RAPPORTÉS}

Si l'EE a été jusqu'à ce jour analysé plutôt à travers l'étude des stratégies argumentatives objectivantes (avec recours à un scripteur universel ou aux évidences perceptuelles, Rabatel 2003c), en revanche, il n'a guère donné lieu à examen dans le champ des discours rapportés (ou représentés, Rabatel 2003a : 74), excepté chez Grossmann 2003 et Rabatel 2003b, en dépit du fait qu'une telle stratégie énonciative ait été explicitement pointée par Charaudeau comme relevant d'une forme d'EE distincte de la première :

Le sujet parlant s'efface de son acte d'énonciation, et n'implique pas l'interlocuteur. Il témoigne de la façon dont les discours du monde (le tiers) s'imposent à lui. Il en résulte une énonciation apparemment objective (au sens de " déliée de la subjectivité du locuteur ») qui laisse apparaître sur la scène de l'acte de communication des Propos et des Textes qui n'appartiennent pas au sujet parlant (point de vue externe).

9. Les analyses de « il me fallait de la menthe » (Haillet 2004 : 12-13) reposent sur une dissociation entre les PDV du locuteur en tant que tel ("moi-maintenant-auteur de l'énoncé ») et du locuteur $\lambda$ (« moi-maintenant-objet du discours »). Une telle disjonction, qui accorde au PDV du locuteur $\lambda$ toute sa place dans l'analyse linguistique, n'est pas sans étayer celle entre actualisations déictique et modale, dans la mesure où c'est la plus ou moins grande saturation du sujet modal qui donne une réalité discursive au locuteur $\lambda$ et/ou à des énonciateurs intratextuels à l'origine de PDV qui ne sont pas des paroles.

10. Illusoire position de maîtrise (comme l'ont montré Freud et Lacan) au demeurant compatible avec l'aliénation sociale et politique des individus... 
Dès lors deux cas peuvent se présenter :

- Le Propos s'impose de lui-même [...]

- Le Propos est un Texte déjà produit par un autre locuteur et le sujet parlant n'aurait donc qu'à jouer un rôle de rapporteur (dont on sait qu'en réalité il peut être plus ou moins objectif. [...] C'est le cas des différentes formes de " Discours rapporté »(Charaudeau 1992 : 649-650).

Le DR, " objectivé » comme " Texte », s'accompagne de formes graduelles $\mathrm{d}^{\prime} \mathrm{EE}$, depuis les manifestations minimales de l'EE, avec la désinscription énonciative (EE lato sensu) jusqu'à l'effacement - idéalement - complet de l'énonciateur (EE stricto sensu). La gradualité du phénomène explique que la désinscription énonciative ne se limite pas aux marqueurs personnels et spatiotemporels, mais qu'elle intègre toute forme de référence dont l'interprétation n'est plus opérée en fonction des données situationnelles ; à ce titre, la désinscription énonciative peut affecter, comme on le verra, ici même, dans les articles de ce numéro,

- le locuteur cité, dont l'origine énonciative est brouillée, voire omise par le locuteur citant, dont les dires initiaux sont re-présentés (et non pas simplement rapportés) sous une forme qui efface une partie de leur contexte initial (îlots textuels, discours indirect ou narrativisé - effaçant le dire originel et les informations véhiculées par ce dire-, discours direct imaginaire, itératif, etc.) ;

- le locuteur citant, qui peut gommer, déplacer, modifier les marques du rapport (suppression du verbe attributif, des marques d'ouverture et de fermeture des dires) ou modifier leur orientation argumentative ; représenter des énoncés originellement embrayés (du moins par convention tacite) comme des énoncés non embrayés, etc.

Ces modifications linguistiques ont des répercussions pragmatiques car elles modifient (à la marge ou en profondeur) le PDV des locuteurs cités, voire opacifient celui du locuteur citant. Elles ne sont pas de même nature, car, en contexte écrit, le locuteur cité est affecté par la stratégie de désinscription énonciative du locuteur citant, tandis que la désinscription du locuteur citant résulte de son choix - ce qui ne préjuge pas de sa pertinence ${ }^{11}$.

Conjoindre l'EE à l'étude des formes de DR ne relève donc pas du paradoxe : en effet, en dépassant le cadre stricto sensu du DR, la démultiplication du dire en circulation brouille ou rend caduque l'identification de la source énonciative parce que :

- le discours en circulation apparaît comme l'expression d'une conscience collective (proverbes, lieux communs, stéréotypes) ;

- seule importe la reconnaissance d'énonciations prototypiques, relevant de genres de discours ou de formations discursives spécifiques (discours des journalistes, discours des politiques, des savants, etc.) ;

11. Ni d'une posture a priori : si le locuteur citant se range à l'avis d'une autorité, la désinscription équivaut à la sousénonciation (Rabatel 2002) ; si en revanche la désinscription vise à imposer à autrui un PDV personnel en le faisant passer pour un PDV " objectif », elle équivaut à la surénonciation ( $c f$. infra, 4). 
- c'est la chaîne de répétition qui prime, au-delà de l'identification énonciative, comme dans la médisance, les potins, les ragots (Rosier et Mailleux 2002).

\section{LES PÔLES DE LA TOPIQUE ÉNONCIATIVE : COÉNONCIATEUR, SURÉNONCIATEUR ET SOUSÉNONCIATEUR}

Les effets pragmatiques qui découlent de l'EE dans les discours citants et cités invitent à proposer des éléments d'une topique énonciative, avec trois postures qui entrent en jeu dans la construction interactionnelle des points de vue (la graphie de coénonciation, sousénonciation, surénonciation suit les préconisations de la réforme de 1990) :

- La coénonciation correspond à la coproduction d'un point de vue commun et partagé. Cette définition la distingue de la coénonciation chez Morel et Danon-Boileau 1998 comme chez Jeanneret 1999 : chez les premiers (comme chez Culioli), la coénonciation ne correspond pas à une coproduction d'un énoncé commun par les deux locuteurs, mais aux calculs du locuteur pour produire un énoncé qui recueille le consensus de l'interlocuteur en anticipant sur ses réactions ; chez la seconde, il s'agit d'un phénomène de colocution qui ne présuppose pas que le point de vue commun soit effectivement partagé par les deux (cf. Rabatel 2004a). Les phénomènes d'accord sur un PDV étant fragiles, limités, la coénonciation s'avère une forme sinon idéale de coopération, du moins très instable, fugace ${ }^{12}$, vite remplacée par la surou la sousénonciation, davantage à même de rendre compte des inégalités, déséquilibres et désaccords qui fleurissent dans la communication.

- La surénonciation ${ }^{13}$ est définie comme l'expression interactionnelle d'un point de vue surplombant dont le caractère dominant est reconnu par les autres énonciateurs.

12. Sauf dans les contextes fusionnels, comme dans les langues de bois, ou dans des communautés discursives fortement soudées par des pratiques langagières communes ( $c f$. les " particitations »), rassemblées en un Thésaurus, ainsi que le montre Maingueneau, ici même. On notera que ces particitations sont, en règle générale, assez peu expansées : l'accord, d'être obtenu en peu de mots, n'en est que plus saillant.

13. La prétention du surénonciateur à voir son PDV repris par les interactants pourrait être mise en parallèle avec ce que Maingueneau et Cossutta écrivent des discours constituants :

«La prétention attachée au discours constituant, c'est de fonder et de n'être pas fondé. Cela ne signifie pas que les multiples autres types d'énonciations (les conversations, la presse, les documents administratifs, etc.) n'ont pas d'action sur eux : bien au contraire, il existe une interaction continuelle entre discours constituants et non-constituants, de même qu'entre discours constituants. Mais il est dans la nature de ces derniers de dénier cette interaction ou de prétendre la soumettre à des principes » (Maingueneau et Cossutta 1995 : 112).

Certes, l'analogie ne doit pas être poussée à son terme, il n'y a rien de commun entre des discours construits, jouant un rôle institutionnel, et l'expression ordinaire de points de vue triviaux. Mais il est intéressant de souligner combien, mutatis mutandis, les locuteurs qui aspirent à l'expression d'un point de vue dominant affichent cette " prétention ", même si dans un cas, elle correspond à une " nature ", dans l'autre à une stratégie, beaucoup plus limitée dans ses effets. 
- La sousénonciation renvoie à l'expression interactionnelle d'un point de vue dominé, au profit d'un surénonciateur ${ }^{14}$.

Co-, sur- et sousénonciation, versus co-, sur- et souslocution

Nous disons bien co-, sur- et sousénonciation, et non pas co-, sur- et souslocution, parce que les relations entre locuteurs ne sont pas directement proportionnelles à la quantité de paroles prononcées : sur ce plan aussi, la déliaison locuteur/énonciateur fait particulièrement sens, dans la mesure où un locuteur peut parler beaucoup sans que son PDV soit interactionnellement dominant. Être surlocuteur n'implique pas qu'on soit surénonciateur. Semblablement, être un petit parleur (quelque chose comme un " souslocuteur ») n'implique pas qu'on soit un sousénonciateur. Il n'est pas nécessaire de parler beaucoup pour $\mathrm{qu}^{\prime}$ un PDV structure les échanges : ainsi, sur un plan situationnel, lorsqu'un chef à la parole rare voit ses subordonnés parler pour lui, se plaçant en sousénonciateurs face au discours de l'autorité, ou lorsque un maître laisse exprimer son PDV par les bons élèves qui devancent ainsi les attentes de l'institution ${ }^{15} \ldots$; ainsi encore, sur un plan générique, lorsque le polémiste cite longuement, pour le discréditer, un PDV adverse, tandis qu'en tant que surénonciateur, il n'a besoin que d'une pointe finale assassine pour exprimer son PDV surplombant.

\section{Une topique}

Il est tentant d'organiser ces postures en une topique qui spatialise les relations entre des notions, à des fins descriptives, à l'instar de la topique de Marx entre infrastructure et superstructure, de celle de Pouillon autour de la théorie littéraire des visions (visions par derrière, avec, $d u$ dehors), qui a inspiré le système des focalisations narratives de Genette, ou des deux topiques freudiennes (inconscient, préconscient et conscient ; ça, moi, surmoi). C'est en ce sens qu'on parle ici de topique énonciative, toute proportion gardée, sans qu'il faille établir de corrélation entre surmoi et surénonciateur, ça et sousénonciateur, ni entre vision par derrière et surénonciation, vision avec et coénonciation, vision du dehors et sousénonciation. Il n'y a rien de commun entre les problèmes d'inégalité dans l'expression des PDV et les instances du psychisme dans le premier cas, le phénomène cognitif et technique de régulation de l'information narrative dans le second, sauf à s'en tenir à une parenté métaphorique sans grand intérêt entre position dominante et position dominée, et à vouloir saturer ontologiquement, psychologiquement ou sociologiquement, une donnée énonciative qui peut certes alimenter ces représentations, en relation avec le système des places, sans pourtant s'y réduire, comme le montre Rosier, ici même.

14. Il est tentant de faire de la surénonciation une surassertion et de la sousénonciation une sousassertion (ou une désassertion). En réalité, les notions ne sont pas du même niveau : surassertion et sousassertion sont des sous-ensembles de la sur- et de la sousénonciation, ces dernières pouvant emprunter d'autres formes que l'assertion ( $c f$. les questions qui équivalent à des " mises en question " analysées par Sitri $2003: 82-99$ ).

15. C'est une autre question que de déterminer si ces sousénonciateurs sont convaincus de ce qu'ils disent, ou jouent la comédie. 


\section{Postures, places et stratégies des locuteurs}

Les postures énonciatives doivent donc être distinguées des places des sujets parlants et des relations entre locuteurs - relations verticale (statut, place) et horizontale (familiarité, distance), relations affectives (attraction, répulsion) et idéologiques (consensus, dissensus). Ainsi, la coénonciation n'implique pas que les locuteurs soient des pairs, partagent les mêmes options intellectuelles ou idéologiques. De même, il n'est pas obligatoire que le surénonciateur corresponde à un statut sociologique ou politique dominant, à une domination intellectuelle : tout comme il est possible de proférer des âneries dans une énonciation théorique, il se peut que tel PDV dominant ne s'avère pas le plus pertinent (Rabatel 2004a). De même pour la sousénonciation. Il faut distinguer ce qui relève d'une posture contrainte ou d'une posture choisie : ainsi, le sousénonciateur peut exprimer un PDV dominé en référence/révérence à une autorité par politesse, respect, admiration, soumission, libre adhésion, aliénation ou feintise, etc. Il serait donc réducteur de réifier les postures en fonction des places ou des stratégies voire des situations. En revanche, la contribution des postures à la dynamique des places est précieuse : en effet, les rapports de co-, sur- et sousénonciation sont surdéterminés par la dialogie interactionnelle et par la situation objective antérieure, mais doivent être vérifiés (ou contestés) au cours même de l'interaction.

\section{Dimension interactionnelle des postures et degrés d'engagement des énonciateurs}

Tout locuteur premier (L1 ou L2) peut adopter toutes les postures de la topique, en contexte dialogal. En contexte monologal, tous les locuteurs enchâssés $(11,12$, etc.) voire même des énonciateurs dans des énoncés délocutés sont des candidats potentiels. Si, à l'oral, la posture résulte du positionnement réciproque des locuteurs dans l'interaction, en revanche, à l'écrit, énonciateur second et locuteur second ne sont sur- ou sousénonciateurs que parce que L1 se positionne lui-même en sous- ou en surénonciateur. Cette différence explique qu'à l'écrit, en contexte monologal, les relations entre sur- et sousénonciateur soient spéculaires. À l'oral, l'indépendance relative des locuteurs/énonciateurs complexifie la donne : ainsi, des locuteurs peuvent adopter une posture de sousénonciation pour discréditer un PDV dominant en le poussant à l'absurde... La dynamique interactionnelle entraîne les locuteurs/énonciateurs à reprendre tel ou tel PDV dominant en l'enrichissant, en le contestant, dans tous les cas en en modifiant la portée, en sorte que le PDV final ne correspond ni à ce que pens(ai)ent les locuteurs/énonciateurs dominés, ni même le locuteur/énonciateur dominant (Rabatel 2002, 2004a, 2004b).

Chaque posture est passible de degrés dans 1'adhésion (ou non) des locuteurs envers les PDV partagés, dominants ou dominés, sur les plans épistémique, scientifique, idéologique, social, politique, éthique, psychologique ou affectif, etc. Sur le plan linguistique, l'analyse des variations dans la prise en charge des énoncés est complexe : outre les éventuelles marques intonatives, syntaxiques ou discursives d'engagement des locuteurs, c'est le déroulement de l'interaction qui indique jusqu'où va l'accord, quand il cesse, au point d'amener à réinterpréter ce qui passait en première analyse pour un accord sur un 
contenu propositionnel comme un accord tacite pour que le PDV fasse l'objet d'un examen sans que l'interlocuteur aille au-delà de cet accord formel (cf. le locuteur vicariant ${ }^{16}$ de Danon-Boileau et Morel 2003).

\section{Structuration discursive et marques linguistiques}

Les postures sont codées en langue et en discours ; toutefois, ces marques sont innombrables, et c'est là une première difficulté. Ainsi, la coénonciation est indiquée par des marques explicites d'accord, tant dans le modus que dans le dictum (ce dernier jouant un rôle crucial avec l'EE, l'accord se faisant sur le dictum, le modus se limitant en général à la reprise de la modalité de phrase, sans commentaires méta-réflexifs) : tout peut faire signe, lexique, syntaxe (reprise de structures syntaxiques, des modalités...), prosodie (effets d'osmose rythmique, intonative), avec un certain nombre de marques spécifiques à l'oral - décondensation du préambule, importance du travail de reformulation, autour des motsoutils, répétés, autour de la dénomination, de la qualification, avec auto-corrections, débit lent (Morel 2003) - sans compter la dimension para-verbale.

La surénonciation se marque dans le DR à travers les choix du locuteur citant de ce qu'il veut rapporter, du moment, des segmentations et des éventuelles transpositions (qui sont loin d'être toutes contraintes), du choix des verba dicendi. Bien d'autres marques entrent en ligne de compte : temps, modalités et modalisations, structures dialogiques - présupposition, négation, reformulation, confirmation, rectification, opposition, renchérissement, discours écho, ironie, discours rapportés, concession, phrase clivée (topicalisation, focalisation), interrogation, question rhétorique, etc. - effets énonciatifs des mises en scène d'acteurs de l'énoncés, du montage et des recontextualisations des citations. Cette énumération témoigne de l'ampleur du problème, ces marques n'étant pas univoques : toutes les marques précédentes $n$ 'indiquent pas nécessairement un surénonciateur pas plus que leur absence ne signale automatiquement un sousénonciateur. La difficulté de systématisation redouble avec la prise en compte des données textuelles, par exemple autour des effets de sur- ou de sousénonciation favorisés respectivement par les schémas déductifs ou abductifs, les descriptions avec ancrage ou affectation, les isochronies ou achronies narratives, le choix de tel point de vue narratif... Autant dire que la tâche est immense et que ce numéro ambitionne seulement de poser des jalons. Quoi qu'il en soit, il paraît trop commode de conclure, au vu de la difficulté de formaliser des marques, que les postures se limitent à des effets interprétatifs ${ }^{17} \ldots$

16. Le locuteur vicariant est celui qui produit une sorte de discours-écho qui ne l'engage pas, mais qui correspond au point de vue du locuteur patenté (Danon-Boileau et Morel 2003 : 242). Selon Morel 2003, les marques de non-engagement envers un pdv sont les suivantes, à l'oral : débit accéléré, absence de décondensation du préambule, absence du travail de formulation syntaxique : syntaxe liée. 


\section{PRÉSENTATION DES ARTICLES}

Les auteurs ont fait le choix délibéré d'inscrire leurs investigations dans le cadre de genres de discours identifiés ${ }^{18}$, dictionnaires ou articles de sciences humaines (Rabatel, Grossmann et Rinck), presse (Marnette, Vion), ou dans celui de pratiques discursives en faveur dans divers milieux (discours puristes chez Rosier, forums chez López Muñoz, énoncés doxiques chez Maingueneau). Ce volume aborde l'ensemble de la topique, y compris la coénonciation (López Muñoz, Maingueneau), mais c'est la surénonciation qui fait l'objet de la plupart des analyses (Rabatel, Grossman et Rinck, Marnette, Rosier), notamment sous l'angle des relations complexes entre surénonciation et sousénonciation (Grossmann et Rinck, Rosier, Marnette, López Muñoz, Vion) ou entre coénonciation et surénonciation (López Muñoz). Dans tous les articles, les effets pragmatiques sont articulés avec des formes variées d'argumentation, qu'il s'agisse des effets argumentatifs résultant de l'argumentation dans la langue, au sens ducrotien, ou des effets argumentatifs indirects reposant sur des schématisations (Grize 1990), sur des topoï qui orientent l'interprétation indépendamment de la présence des formes argumentatives « logiques »(Amossy 2000).

À partir d'un exemple de dictionnaire philosophique, Alain Rabatel étudie la construction du surénonciateur à travers des formes d'effacement énonciatif stricto et lato sensu, en s'attachant d'abord, sur le plan discursif, à diverses stratégies de mise en scène énonciative, ensuite, sur le plan des énoncés, aux indices de surénonciation apparaissant lors de la recontextualisation des paroles rapportées (choix du matériel cité, des bornes, sélection et choix de tiroir des verba dicendi, modification de portée et des modalités des propos originels, etc.) ainsi qu'à la mise en jeu de mécanismes d'argumentation par autorité polyphonique. Grâce à ces stratégies d'effacement de son point de vue personnel, le locuteur /énonciateur premier parvient à imposer sans contestation un point de vue apparemment « objectif » et « naturel » (le sien), en se faisant passer pour l'alter ego de ses pairs, voire, du fait de sa posture de surénonciateur, de primus inter pares.

Francis Grossmann et Fanny Rinck analysent des dictionnaires et des articles de linguistique dans lesquels la surénonciation est une posture obligée. Les instances de prise en charge énonciative y obéissent à un principe de cohérence polyphonique permettant leur regroupement et leur indexation - certes provisoire -

\footnotetext{
.../... Comme l'archi-interprétant, la surinterprétation (en relation avec le malentendu, lorsque « le destinataire voit une valeur indirecte là où le locuteur prétendait parler directement ») et la sous-interprétation (en relation avec la mauvaise foi, lorsque "le destinataire ne perçoit pas, ou feint de ne pas percevoir, la valeur dérivée ») (Kerbrat-Orecchioni, in Charaudeau et Maingueneau 2002 : 20), méritent d'être confrontés à la topique, même s'il faut se garder d'assimiler la surinterprétation à la surénonciation et la sousinterprétation à la sousénonciation, car implicite et EE ne se recouvrent pas. Ces relations feront l'objet d'un traitement spécifique dans le $\mathrm{n}^{\circ} 22$ de Semen (Rabatel A. et Chauvin-Viléno A., éds), à paraître en 2006, à propos de la presse, et dans Lidil, à propos de contextes didactiques écrits et oraux (Rabatel A. et Grossmann F., éds), à paraître en 2007.
}

18. Cf. Bouquet 2004, Langages 153. 
au locuteur, tout en maintenant leur hétérogénéité. D'où l'intérêt qu'il peut y avoir à relier la question de la prise en charge énonciative et celle, plus externe, d'auctorialité. Dans ce cadre, la surénonciation joue un rôle argumentatif de légitimation, se manifestant sous des formes très diverses. Dans les dictionnaires, la surénonciation dépend des possibilités structurelles du genre et d'une gestion uniformisante de la polyphonie, qui masque l'hétérogénéité des référents théoriques et l'ambivalence du statut assertif des énoncés. Dans les articles, elle repose plus nettement sur la pluralité des énonciateurs, le dialogisme servant à légitimer une instance auctoriale qui se présente comme décentrée, parfois clivée.

Sophie Marnette traite de l'effacement énonciatif dans la presse contemporaine. Son enquête envisage les liens entre effacement énonciatif et discours rapporté dans la pratique journalistique en se basant sur un corpus diversifié incluant magazines féminins, magazines de la presse " people ", magazines d'information et quotidiens. Sophie Marnette analyse d'abord des cas où l'emploi du discours rapporté met clairement le journaliste en position de surénonciation, par ses commentaires sur son discours ou sur celui des locuteurs cités, par l'effacement de l'origine des locuteurs cités, par les recontextualisations des dires. Dans un deuxième temps, elle met en relief des situations plus complexes où le journaliste joue sur les deux postures de surénonciation et de sousénonciation, en s'effaçant derrière les sources cités, tout en les mettant en scène en fonction de son point de vue (ou de celui de son journal), en sorte qu'une stratégie peut en cacher une autre.

Laurence Rosier part d'une tentative de formalisation de la notion de circulation des discours. Cette problématique oblige à s'intéresser à l'origine et aux postures énonciatives prises dans le discours (les phénomènes de sousénonciation, de surénonciation, de travestissements énonciatifs) ainsi qu'à ses mécanismes de diffusion et de réception. Redéfinissant ces trois notions selon la légitimité discursive du locuteur et sa mention (ou son absence de mention) comme énonciateur dans le discours citant, Laurence Rosier décrit quelques pratiques " rusées » de travestissements énonciatifs comme le pseudonyme, et met à l'épreuve de ses propositions théoriques certaines manifestations du discours puriste sur la langue (dictionnaires de critique ironique de la langue, chroniques de langues), qui privilégient des mécanismes de sousénonciation, aux frontières de l'effacement énonciatif.

Juan Manuel López Muñoz aborde la question de l'effacement énonciatif dans le discours rapporté en lien avec la problématique de co-construction de l'opinion, par le biais des discours des autres, dans les forums qui ont cours sur le site du journal Le Monde ; cette co-construction, qui ne produit pas une opinion collective unanime, est appréhendée à partir des paradigmes de la pragmatique et analysée dans ses dimensions énonciative, interactionnelle et argumentative. Son hypothèse est que l'EE repose sur la base des connaissances métapragmatiques et que c'est sur ce socle qu'il acquiert son pouvoir d'argumentation. Juan Manuel López Muñoz montre l'importance des stratégies argumentatives de contre-argumentation, produisant un effet de surénonciation, dans les cas d'extraction, et de justification, avec une coénonciation à visée surplombante, dans les cas où le locuteur recourt à un point de vue indéterminé. 
Robert Vion analyse les postures de surénonciation et de sousénonciation en prolongeant ses travaux antérieurs sur la modalité et la modalisation, en lien avec la notion de mise en scène énonciative, qui s'avèrent des outils précieux pour une analyse du dynamisme discursif. La modalité est définie comme une réaction du locuteur vis-à-vis d'une représentation qu'il construit lui-même dans son discours. Cette réaction, qui se traduit par l'inscription de la représentation dans un univers particulier, $\mathrm{n}^{\prime}$ a que peu de choses à voir avec la modalisation qui repose sur un dédoublement énonciatif avec production d'un commentaire réflexif. Autant la modalité contribue au sens de l'énoncé, autant la modalisation complexifie la mise en scène au point d'opacifier le sens et de brouiller la relation. Partant, Robert Vion examine la manière dont fonctionne le déroulement discursif en croisant ces outils avec la prise en compte de la parole représentée et des phénomènes de sur- et de sousénonciation.

Dominique Maingueneau introduit une nouvelle instance énonciative à propos de citations qui ne mentionnent pas leur auteur : "l'hyperénonciateur ». Ces citations (nommées " particitation », terme valise qui associe «participation » et " citation ») relèvent d'un régime de citation spécifique, concernant des énoncés autonomes qui font partie d'un Thésaurus dont la reconnaissance présuppose la co-appartenance du locuteur et de l'allocutaire à une même communauté, ce qui explique que la coénonciation opère sans marquage explicite d'accord. Le locuteur est censé citer ce qui pourrait/devrait être dit par l'allocutaire - et au-delà tout membre de la communauté qui agit de manière pleinement conforme à cette appartenance -, l'« hyperénonciateur ", garant de ce Thésaurus, se voyant mobilisé dans la prise en charge de l'énoncé. Grâce à cette caractérisation première, divers types de particitations sont distingués (particitations sentencieuses, scripturaires, de groupe), défrichant un champ dont le découpage implique une perspective anthropolinguistique.

Ainsi, toutes les contributions de ce numéro de Langages abordent à des titres divers la question des effets pragmatiques de l'EE, interrogation qui n'est pas sans conséquence pour l'analyse linguistique comme pour celle de la circulation des idéologies, de part en part traversées par le dialogisme, y compris dans les processus d'objectivation sous lesquels elles se reproduisent ${ }^{19}$.

19. Cf. ces propos de Grunig :

«Les processus de transmission non déclarée ont été infiniment moins pris en compte [que les processus de transmission déclarée], ne serait-ce qu'à cause de l'absence même d'indice signifiant la transmission. Un sujet locuteur dit :

- Le directeur ne viendra pas

ou

- Les paysans ont bloqué la place de la Concorde

et rien n'indique spécifiquement dans son propos qu'il le tient (forme et/ou contenu) d'un locuteur antérieur. Il redit simplement ce qui lui est parvenu. On peut certes, en tant que linguiste attentif au signifiant, ne pas vouloir s'attarder sur ces transmissions non déclarées, mais il me semble que c'est une obligation intellectuelle - et parfois morale - de ne pas laisser ignorer la portée immense de ce phénomène. Songeons en effet à tout ce que nous recevons et " passons au suivant ", comme un ballon, en omettant la mention d'origine, et d'ailleurs, souvent, en n'ayant plus aucune conscience nette d'une origine »(Grunig 1994 : 129). 


\section{Références bibliographiques}

Amossy Ruth, 2000, L'argumentation dans le discours. Nathan, Paris.

AUTHIER-REVUz Jacqueline, 1995, Ces mots qui ne vont pas de soi. Boucles réflexives et noncoïncidences du dire. T. 1 et 2. Larousse, Paris.

AUtHIER-RevUz Jacqueline, 1998, "Énonciation, méta-énonciation. Hétérogénéités énonciatives et problématiques du sujet ", in Vion, R. (éd), Les sujets et leurs discours. Énonciation et interactions, 63-79. Publications de l'Université de Provence, Aix-enProvence.

BENVENISTE Émile, 1966, Problèmes de Linguistique générale, 1. Gallimard, Paris.

Benveniste Émile, 1974, Problèmes de Linguistique générale, 2. Gallimard, Paris.

BOUQUET Simon (dir), 2004, Les genres de la parole. Langages 153.

Boch Françoise et Grossmann Francis, 2002, "Se référer au discours d'autrui, quelques éléments de comparaison entre experts et néophytes "Enjeux 54, 41-51.

Bres Jacques, 2001, Article "Dialogisme ", in Détrie, C. Siblot, P. et Verine, B. (éds) Termes et concepts pour l'analyse du discours. Champion, Paris.

Charaudeau Patrick, 1992, Grammaire du sens et de l'expression. Hachette, Paris.

Charaudeau Patrick et Maingueneau Dominique (dir), 2002, Dictionnaire d'analyse du discours. Le Seuil, Paris.

CoHn Dorrit, 1981 [1978], La transparence intérieure. Le Seuil, Paris.

Danon-BoIleau Laurent et MoRel Mary-Annick, 2003, " Le locuteur vicariant ", in Merle, J.-M. (éd), Le sujet, 235-246. Ophrys, Gap, Paris.

DÉtRIE Catherine, Siblot Paul, Verine Bertrand (dir), 2001, Termes et concepts pour l'analyse du discours. Une approche praxématique. Champion, Paris.

Ducrot Oswald, 1984, Le dire et le dit. Minuit, Paris.

Ducrot Oswald, 1993, "À quoi sert le concept de modalité ? " in Dittmar, N. \& Reich, A. (éds) Modalité et acquisition des Langues, 11-129. Walter de Gruyter, Berlin.

GofFMAN, Erving 1987 [1981], Façons de parler. Minuit, Paris.

Grossmann Francis, 2002, "Les modes de référence à autrui chez les experts : l'exemple de la revue Langages ", in Faits de Langues 19, 255-261.

Grossmann Francis, 2003, "Du discours rapporté au discours autorisé, le maniement des noms d'auteur dans l'article en Sciences Humaines ", Estudios de Lengua y literatura francesas, 14, 9-31. Université de Cadix.

GRIZE Jean-Blaise, 1990, Logique et langage. Ophrys, Gap, Paris.

GRUNIG Blanche-Noëlle, 1994, "Pour une conception dynamique du sujet ", in Yaguello, M. (éd), Subjecthood and Subjectivity. The status of the subject in linguistic theory, 125137. Ophrys, Gap, Paris.

HAILlet Pierre Patrick, 2004, "Nature et fonction des représentations discursives : le cas de la stratégie de la version bémolisée ", Langue Française 142, 7-16.

JEANNERET Thérèse, 1999, La coénonciation en français. Peter Lang, Berne.

JouvE Vincent, 1992, L'effet-personnage dans le roman. Presses Universitaires de France, Paris.

Kerbrat-Orecchioni Catherine, 2002, "Rhétorique et interaction ", in R. Koren et R. Amossy (éds.), Après Perelman : quelles politiques pour les nouvelles rhétoriques ?, 173-196. L'Harmattan, Paris.

Lopez Muñoz Juan Manuel, MARnetTe Sophie et Rosier Laurence (éds), 2002, Le discours rapporté. Faits de langues 19.

LoPeZ MuÑoz Juan Manuel, MARNETTE Sophie et Rosier Laurence (éds), 2004, Le discours rapporté dans tous ses états. L'Harmattan, Paris.

Maingueneau Dominique, 1990, Pragmatique pour le texte littéraire. Bordas, Paris.

MaIngueneau Dominique et Cossutta Frédéric, 1995, "L'analyse des discours constituants ", Langages 117, 112-125.

Morel Mary-Annick et DANON-BOILEAU Laurent, 1998, Grammaire de l'intonation. L'exemple du français. Ophrys, Gap, Paris. 
Morel Mary-Annick, 2003, "Fusion/dissociation des points de vue dans le dialogue oral : intonation et syntaxe discursive ", Cahiers de praxématique 41, 157-189.

NøLKE Henning, 2002, Article "Polyphonie ", in Charaudeau, P. et Maingueneau, D. (éds) Dictionnaire d'analyse du discours. Le Seuil, Paris.

NøLKE Henning et OLSEN Michel, 2000, "Polyphonie : théorie et terminologie ", Polyphonie linguistique et littéraire 2, 45-171. RUC (Working Paper), Université de Roskilde, Danemark.

PHILIPPE Gilles, 2002, " L'appareil formel de l'effacement énonciatif et la pragmatique des textes sans locuteur " in Amossy, R. (éd), Pragmatique et analyse des textes, 17-34. Université de Tel-Aviv.

PoullLon Jean, 1993 [1946], Temps et roman. Gallimard, Paris.

RABATEL Alain, 1997, Une histoire du point de vue. Klincksieck, Université de Metz, Paris, Metz.

RABATEL Alain, 2002, "Le sous-énonciateur dans les montages citationnels : hétérogénéités énonciatives et déficits épistémiques ", Enjeux 54, 52-66.

RABATEL Alain 2003a " Les verbes de perception en contexte d'effacement énonciatif : du point de vue représenté aux discours représentés ", Travaux de linguistique 46-1, 49-88.

RABATEL Alain, 2003b, " L'effacement énonciatif et ses effets pragmatiques de sous- et de sur-énonciation ", Estudios de Lengua y Literatura francesas 14, 33-61. Université de Cadix.

RABATEL Alain, 2003c, "Un paradoxe énonciatif : la connotation autonymique représentée dans les 'phrases sans parole' stéréotypées du récit ", in Authier-Revuz, J., Doury, M. et Reboul-Touré, S. (éds), Parler des mots. Le fait autonymique en discours, 271-280. Presses de la Sorbonne Nouvelle, Paris.

RABATEL Alain, 2003d, " Le point de vue, entre langue et discours, description et interprétation : état de l'art et perspectives ", Cahiers de Praxématique 41, 7-23.

RABATEL Alain, 2004a, "Déséquilibres interactionnels et cognitifs, postures énonciatives et co-construction des savoirs : co-énonciateurs, sur-énonciateurs et archi-énonciateurs ", in Rabatel, A. (éd), Interactions orales en contexte didactique. Mieux (se) comprendre pour mieux (se) parler et pour mieux (s')apprendre, 26-69. Presses Universitaires de Lyon, Lyon.

RABATEL Alain, 2004b, " La narrativisation d'un texte argumentatif : résolution des conflits et argumentation propositive indirecte ", in Bouchard, R. et Mondada L. (éds). La rédaction collaborative. L'Harmattan, Paris.

RABATEL Alain, 2004c, "La part de l'énonciateur dans la construction interactionnelle des points de vue ", Marges linguistiques 8.

Rosien Laurence, 1999, Le discours rapporté. Histoire, théories, pratiques. Duculot, Bruxelles.

Rosier Laurence et MAILleux Catherine, 2002, " Dire du mal de : étude linguistique d'une énonciation médisante ", Faits de Langues 19, 245-254.

SITRI Frédérique, 2003, L'objet du débat. La construction des objets de discours dans des situations argumentatives orales. Presses de la Sorbonne Nouvelle, Paris.

VION Robert, 1998, "Du sujet en linguistique ", in Vion R. (éd.), Les sujets et leurs discours. Énonciation et interaction, 189-202. Publications de I'Université de Provence.

VION Robert, 2001, "'Effacement énonciatif' et stratégies discursives ", in De la syntaxe à la narratologie énonciative, De Mattia, M. et Joly, A. (éds), 331-354. Ophrys, Gap, Paris. 